\title{
Errore e bioetica nefrologica
}

\author{
Mario Timio $^{1,2}$, Francesca Timio ${ }^{1}$ \\ ${ }^{1}$ Dipartimento di Medicina Interna, Nefrologia e Dialisi, Ospedale di Foligno, Foligno (PG) \\ ${ }^{2}$ Direzione Scientifica Centro di Bioetica della Regione Umbria, Perugia
}

\begin{abstract}
Mistakes and bioethics in nephrology
"Acetate intolerance" was one of the first negative signs of acetate hemodialysis registered in the history of the treatment of chronic renal failure. Now we consider an error such an approach to kidney disease. But it was an "institutional" error according to the classification of the Italian historian of Medicine, Ettore De Benedetti, to distinguish it from the "personal" error, committed after the revision of acetate treatment and the introduction of bicarbonate dialysis. The same difference could be considered in the "short hemodialysis" technique for the adverse cardiovascular outcome. Many examples of "institutional" and "personal" errors in nephrology are reported. Errors need to be recorded and to be analysed if we want to discover why they occur and how they must be prevented. If we want to eliminate errors, it is mandatory to overcome certain attitudes, deeply rooted in the profession. Medical attitudes are often determined by medical bioethics and by the principles that determine whether ideas or actions are considered right or wrong. Errors by doctors are common. In order to reduce errors many authorities tried to reduce them giving some methodological advices. Very important are the proposals of a clinician, Neil Mclntyre, and a philosopher, Karl Popper, for a "new" professional ethics summarized in ten theses, reported in this article.
\end{abstract}

Keywords: Institutional and personal mistakes, New bioethics

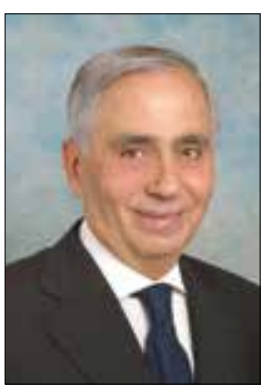

Mario Timio

\section{Errori della medicina ed errori del medico}

Fino agli anni '80, il tampone acetato di sodio era prevalente nella pratica emodialitica. Veniva utilizzato tale tampone poiché la sostanza ha un basso peso molecolare e un rapido metabolismo e favorisce la permanenza in soluzione degli ioni $\mathrm{Ca}++$ e $\mathrm{Mg++}$. Però, è dotato di numerosi effetti clinici negativi che superano quelli positivi: instabilità cardiovascolare intradialitica, vasodilatazione, depressione miocardica con sviluppo di aritmie, tendenza all'iperlipidemia con accelerazione dell'aterosclerosi, ipossiemia e attivazione dell'interleuchina 1 corresponsabile della vasodilatazione (1).

Accepted: April 20, 2016

Published online: May 25, 2016

Indirizzo per la corrispondenza:

Prof. Mario Timio

Via XX Settembre 22

06100 Perugia

timma@libero.it
Questi effetti collaterali hanno indotto a utilizzare il bicarbonato di sodio come tampone, oggi di uso universale, essenzialmente per la maggiore stabilità cardiaca e per la riduzione degli episodi ipotensivi durante l'emodialisi. La conseguente maggiore efficienza dialitica si traduce in una riduzione di morbilità e mortalità (2). Sempre intorno agli anni '80, per un certo periodo, andava di moda l'emodialisi breve (circa due ore), utilizzando l'emodiafiltrazione ad alto flusso con il presupposto che la brevità comportasse miglioramenti emodinamici e cardiaci (3). La tecnica opposta a quella di Tassin, o dialisi lunga (8 ore), con il tempo si è dimostrata negativa per l'apparato cardiovascolare, essenzialmente per lo stress emodinamico, che comportava un aggravamento o l'insorgenza di patologie del cuore e dei vasi, annullando il probabile vantaggio per il paziente di ridurre di circa la metà la durata della seduta. Oggi, di fatto, è una tecnica abbandonata. Che cosa insegnano queste due "narrative" al fine della tematica che stiamo iniziando a trattare, l'errore in medicina? Chi utilizzava la tecnica emodialitica con tampone acetato di sodio o l'emodialisi breve commetteva un errore alla luce dei successivi progressi scientifici. Ma che tipo di errore era? Secondo le acute argomentazioni di Enrico Poli, illustre clinico medico milanese, bisogna distinguere "gli errori derivanti dall'impiego di una cattiva teoria da quelli dipendenti dal cattivo uso di teorie intrinsecamente esatte" (4). Questa distinzione rimanda a quella delineata da Ettore De Benedet- 
ti, medico e storico della medicina, secondo il quale si deve parlare di errori della medicina (istituzionali) e di errori o, meglio, di sbagli del medico (o personali) (5). I primi sono ascrivibili esclusivamente alle lacune e all'insufficienza delle conoscenze mediche del momento, mentre i secondi sono determinati dall'incapacità del medico a giungere a una diagnosi o a un cura giusta sulla scorta delle nozioni all'epoca note. Un'esemplificazione storica dell'errore in medicina, o errore istituzionale, è quella del salasso, una pratica medica che, pur resistendo nel tempo, ha creato più danni che vantaggi. Perché ha creato danni? Perché all'epoca si credeva che la quantità di sangue circolante fosse pari a 25-30 litri, per cui un salasso di 4-5 litri era la regola. Ma questa regola "istituzionale" poteva risultare fatale, giacché, di fatto, si sottraeva quasi tutto il contenuto sanguigno circolante, che, in realtà, è pari a 4-5 litri. Allora, non è senza fondamento l'affermazione che a Parigi ha procurato più decessi il salasso che la ghigliottina durante la rivoluzione francese (6). E ciò per un errore della medicina, un errore attribuibile alla grammatica di lettura costituita dalle teorie del tempo.

Scendendo nei particolari emodialitici, è un errore istituzionale della medicina l'iniziale dialisi con acetato, poiché non si conoscevano ancora gli effetti cardiovascolari connessi alla tecnica. Se oggi si usa ancora l'acetato siamo di fronte a un errore o, meglio, a uno sbaglio del medico. Lo stesso discorso vale per l'emodialisi breve o ultrabreve. Errore della medicina prima, errore del medico dopo che la Medicina Basata sulle Evidenze (EBM) ha fatto emergere gli aspetti negativi cardiovascolari della tecnica ad alti flussi. Ancora una volta lo stesso errore prima è inevitabile, poi è evitabile, prima è istituzionale e, poi, è personale. Quindi, è un errore crono-dipendente.

\section{Errore come infrazione del metodo: anche i luminari sbagliano}

Esemplificazioni dello sbaglio medico sono quelle legate a fattori intrinseci come l'ignoranza, la disattenzione, la trascuratezza, l'imperizia, l'imprudenza, la suggestione, l'incuria e la presunzione. Tutti fattori validi ma non esaustivi. L'errore del medico è un'infrazione al metodo, cioè al procedimento diagnostico e curativo. Ed è proprio il metodo, ovvero la metodologia, a fornire la bussola che conduce al procedimento diagnostico e curativo corretto. Esempi di errori del medico o di sbagli sono infiniti. Ognuno di noi è depositario di tante infrazioni del metodo, personali e di altri. Ne cito uno di molti anni fa, noto per avere impressionato l'opinione pubblica internazionale. Mi riferisco alla mancata diagnosi di malaria che ha condotto allo morte Fausto Coppi, campione di ciclismo. Era il 1960. Un grande errore medico, un grande sbaglio. La storia in sintesi. Il campione si reca in Africa Centrale per una partita di caccia. Dopo alcuni giorni accusa malessere, stanchezza e modesta elevazione febbrile. Ritorna in Italia e viene ricoverato per una crisi febbrile che recede dopo 24 ore per ripresentarsi in modo intermittente il giorno successivo. Si attivano i test clinici, laboratoristici e strumentali. È presente una splenomegalia. Una rx-torace conferma la presenza di broncopolmonite e si inizia una terapia antibiotica. Coppi presenta ancora un attacco iperpiretico. Si insiste con gli antibiotici anche su consiglio di un luminare della medicina. Gli accessi continuano. Durante uno di questi, il campionissimo decede. Un'ora prima di morire è stato effettuato un esame del sangue periferico su striscio sottile, che ha evidenziato la presenza del plasmodium falciparum, agente eziologico della malaria estiva autunnale. Troppo tardi. Una compressa di chinino lo avrebbe salvato (7). Dove è stata infranta la regola del metodo diagnostico e curativo nella malattia di Coppi? II non aver considerato la provenienza da una zona endemica malarica, quale può essere l'Africa Centrale, è il primo errore metodologico commesso dal medico. Non sono state, cioè, emesse ipotesi "geografiche" che avrebbero aiutato a formulare la diagnosi. II secondo errore è logico, nel senso che, pur concedendo che la febbre provenisse dal focolaio broncopneumonico, non sono stati tenuti nel debito conto il tipo intermittente degli accessi febbrili, la splenomegalia che progrediva di pari passo con il ripetersi delle crisi iperpiretiche e la loro periodicità. Questa scorrettezza logica ha impedito l'unico tempestivo approccio diagnostico dirimente: la ricerca del plasmodio nel sangue. Quindi, si è attivato il tandem: infrazione metodologica-infrazione logica. E i nefrologi possono incappare in errori quando utilizzano farmaci? Nel mieloma multiplo, prima della terapia con trapianto di cellule staminali ematopoietiche autologhe, sono previsti cicli di autoinduzione con talidomide. I meno giovani ricordano che il farmaco somministrato come psicofarmaco sedativo in donne gravide negli anni '60 è stato associato allo sviluppo di una focomelia nei neonati (8). $E$, quindi, è stato ritirato dal mercato. Per poi ricomparire come immunomodulatore utilizzabile in malattie cutanee e renali. E se, domani, compare qualche anomalia somatica o genetica, commette il medico un errore? Forse sì, ma di tipo istituzionale o errore della medicina, come già lo abbiamo etichettato. E come lo abbiamo attribuite a particolari patologie umane. Oggi, però, abbiamo il conforto delle Linee Guida e del "Vangelo" dell'EBM. Dovrebbero aiutare il medico a non sbagliare. Non tutti non sono d'accordo. Uno è Giuseppe Bianchi, illustre nefrologo e ipertensiologo milanese, che esprime perplessità nell'applicare ai singoli pazienti le conoscenze scientifiche basate sull'EBM ottenute notoriamente in gruppi di pazienti eterogenei non solo sotto il profilo clinico e antropologico, ma, soprattutto, sotto l'aspetto genetico, che rende il paziente unico nelle sue peculiarità e specificità (9). Particolari questi che modulano non solo le caratteristiche cliniche ma anche la risposta farmacologica e che spesso fanno saltare anche le Linee Guida più corrette e di maggiore peso scientifico. $\mathrm{E}$ che possono mettere in difficoltà il medico che deve scegliere, per non 
incorrere in errori che, in questi casi, avrebbero una doppia valenza: istituzionali e personali, aprendo scenari culturali che mai si sono verificati nella storia della medicina. Come fa oggi il medico a tradurre in operatività clinica, immune da errori, Linee Guida EBM dipendenti viziate da basi confuse? Come deve orientarsi il nefrologo, per non commettere errori, di fronte alla nefropatia membranosa, quando sa, per penna di eminenti colleghi come Ponticelli, che la stessa terapia (cortisonici e immunosoppressori) ha tre contrastanti outcome: 1) inefficacia, 2) efficacia incerta e 3) efficacia (10)?

\section{Dieci punti per sbagliare di meno}

Sull'errore del medico si è detto tutto da parte di tutti. Persino filosofi ed epistemologi auspicano una nuova etica in medicina. In fin dei conti, la bioetica "laica" del medico è il rispetto dell'uomo sgravato da errori. In quale modo le aspettative bioetiche del paziente sono rispettate? L'epistemologo e filosofo Karl Popper, che abbiamo ripetutamente citato in altri articoli di questa rubrica, risponde così: "Gli errori dei medici sono comuni. Alcuni errori sono incolpevoli e inevitabili, altri sono colpevoli. Qualunque sia il carattere dell'errore, è, ovviamente, responsabilità dei medici tentare di ridurre la probabilità di errore. Ma, benché $i$ medici possano riconoscere questa responsabilità in linea di principio, vi sono poche prove che essi spendano molto tempo ad analizzare i loro errori nella pratica clinica e, addirittura, durante il loro tirocinio" (11). Anche se non condivisibile in ogni sua parte, il pensiero del filosofo, non medico, ci ricorda che l'errore è un processo biologico fondamentale: è la base di ogni apprendimento, del "trarre profitto dall'esperienza", dell' "imparare dai propri sbagli". Per facilitare l'approccio alla revisione dell'errore per trarne conseguenze positive per i malati, lo stesso Popper propone, insieme al clinico Mc Intyre, una nuova etica professionale su una rivista medica, British Medical Journal, articolata in dieci punti così sintetizzati:

1) La nostra attuale conoscenza congetturale supera di gran lunga ciò che ogni persona può conoscere.

2) Siamo tutti fallibili ed è impossibile per chiunque evitare di commettere errori, anche quelli evitabili.

3) Tuttavia, rimane nostro compito evitare gli errori.

4) Gli errori possono nascondersi anche nelle nostre teorie controllate meglio.

5) Per tutte queste ragioni, il nostro atteggiamento nei confronti dell'errore deve cambiare. $\grave{E}$ proprio qui che deve iniziare la riforma etica.

6) II nostro nuovo principio deve essere quello di imparare dai nostri errori, in modo tale da evitarli in futuro; questo dovrebbe avere la precedenza anche sull'acquisizione di nuove informazioni.

7) $\grave{E}$, quindi, nostro compito cercare i nostri errori e analiz- zarli in maniera esaustiva. Dobbiamo abituarci a essere autocritici.

8) Dobbiamo riconoscere che l'autocritica è la cosa migliore, ma che la critica da parte di altri è necessaria e particolarmente preziosa se essi affrontano i problemi da una prospettiva differente.

9) Se siamo noi ad attirare l'attenzione degli altri sui loro errori, dovremmo ricordare a noi stessi errori simili commessi da altri. Dovremmo ricordare che errare è umano e che persino i grandi scienziati e clinici hanno commesso errori.

10) La critica razionale dovrebbe essere diretta verso errori precisi, chiaramente individuati. Dovrebbe essere espressa in una forma che permetta la sua confutazione (12).

Per la rifondazione della bioetica formulata da un filosofo non è male.

\section{Conclusioni}

Da questo excursus si comprende come il nefrologo, al pari di tutti gli altri medici, è sempre esposto all'insidia dell'errore. Egli si rende conto che non c'è nessun metodo che lo renda totalmente immune. Dell'errore, però, può fare tesoro, per essere guidato verso una diagnosi e una cura migliori. Ma ciò avviene se egli è educato fin dagli studi universitari allo spirito critico e alla formazione di un retto criterio metodologico. Dice Murri, clinico Medico a Bologna, che "per la formazione di un retto criterio medico, sarebbe di beneficio incalcolabile una cattedra degli errori medici: I'esame critico di questi errori costituirebbe il più utile insegnamento di logica medica; si vedrebbe, così, che ognuno si fa la logica da sé e si vedrebbe anche che uno se la fa bene e uno se la fa male, il che non è perfettamente la stessa cosa" (13). C'è sempre una componente pedagogica nell'errore medico. Sottolinea ancora il clinico bolognese: "Ogni giorno si corregge un errore, ogni giorno si corregge una verità, ogni giorno si impara a sapere meglio quello che possiamo fare di bene e quello che siamo condannati ancora a lasciare avvenire di male, ogni giorno erriamo meno della vigilia e impariamo a sperare di fare meglio la dimane". Soltanto dalla critica e dalla più minuziosa ricerca dell'errore si avrà la più efficace profilassi dell'errore. La nuova bioetica in senso popperiano, come caso particolare della metodologia clinica, è tutta qui.

\section{Disclosures}

Financial support: No financial support was received for this submission.

Conflict of interest: The authors have no conflict of interest. 


\section{Bibliografia}

1. IsekiK,OnoyamaK, MaedaT,etal.Comparison of hemodynamics induced by a conventional acetate hemodialysis, bicarbonate hemodialysis and hemofiltration. Clin Nephrol 1980;14: 294-8.

2. Zucchelli P. Apparato cardiovascolare e bicarbonato dialisi. Atti: Il Cuore nelle Nefropatie e nella Dialisi. Assisi 1989;507-15.

3. Cioni L. L'emofiltrazione ad alto flusso: vantaggi e limiti. Atti: II Cuore nelle Nefropatie e nella Dialisi. Assisi 1989;491-7.

4. Poli E. Metodologia medica. Principi di logica e pratica clinica. Rizzoli, Milano 1966; $2^{\circ}$ ed.: 156-7.

5. De Benedetti E. Medicina allo specchio. Edizioni Vitalità Saluzzo 1967;119-42.
6. Timio M. Significato epistemologico degli errori medici. Working Paper. LUISS, Roma 1995;3.

7. Timio M. Ibidem;4-5.

8. Sjostrom H, Nilsson R. II Talidomide e il potere dell'industria farmaceutica. Feltrinelli, Milano 1973;I-XXVII.

9. Bianchi G. Evidence-based medicine. Current opinion in nephrology and hypertension. 1998;7:185-7.

10. Ponticelli C. Le Linee Guida della SIN. G Ital Nefrol 1999;16:1-10.

11. Popper K. L'atteggiamento critico in medicina. La necessità di una nuova etica. In: Popper K. Dopo la società aperta. Armando Editore, Roma 2000;463-79.

12. Mc Intyre N, Popper K. The critical attitude in medicine: the need of a new ethics. Brit Med J 1983; 287:1919-23.

13. Murri A. Quattro lezioni e una perizia. Zanichelli, Bologna 1972. 